\title{
A!
}

This is an electronic reprint of the original article.

This reprint may differ from the original in pagination and typographic detail.

Pöykkö, S.; Puska, M.J.; Nieminen, R.M.

\section{Chlorine-impurity-related defects in $\mathrm{ZnSe}$}

Published in:

Physical Review B

DOI:

10.1103/PhysRevB.57.12164

Published: 15/05/1998

Document Version

Publisher's PDF, also known as Version of record

Please cite the original version:

Pöykkö, S., Puska, M. J., \& Nieminen, R. M. (1998). Chlorine-impurity-related defects in ZnSe. Physical Review B, 57(19), 12164-12168. https://doi.org/10.1103/PhysRevB.57.12164

This material is protected by copyright and other intellectual property rights, and duplication or sale of all or part of any of the repository collections is not permitted, except that material may be duplicated by you for your research use or educational purposes in electronic or print form. You must obtain permission for any other use. Electronic or print copies may not be offered, whether for sale or otherwise to anyone who is not an authorised user. 


\title{
Chlorine-impurity-related defects in $\mathrm{ZnSe}$
}

\author{
S. Pöykkö, ${ }^{*}$ M. J. Puska, ${ }^{\dagger}$ and R. M. Nieminen ${ }^{\ddagger}$ \\ Laboratory of Physics, Helsinki University of Technology, Helsinki FIN-02015 HUT, Finland
}

(Received 15 October 1997)

\begin{abstract}
Defect complexes formed by chlorine-impurity atoms and native defects in $\mathrm{ZnSe}$ are studied by firstprinciples electronic-structure calculations. The strong tendency for the formation of vacancy-impurity pairs is shown. The chlorine-impurity-zinc-vacancy complex is shown to be the most important source of donor compensation. The results presented are compared with recent experimental results. [S0163-1829(98)02519-3]
\end{abstract}

\section{INTRODUCTION}

The wide-band-gap semiconductor $\mathrm{ZnSe}$ has received considerable attention during the last ten years. The large and direct band gap makes $\mathrm{ZnSe}$ a potential material for many optoelectronic applications. Since $p-n$ junctions are needed for most of these applications, controllable doping methods to achieve both $n$ - and $p$-type material are required. For $\mathrm{ZnSe}$, an effective $p$-type doping has been extremely difficult to obtain. The highest hole concentrations have been achieved by the incorporation of active atomic nitrogen. ${ }^{1,2}$ Chlorine has turned out to be the most successful $n$-type dopant in $\mathrm{ZnSe}$. The net donor concentration has been found to increase linearly with the incorporated chlorine concentration up to $10^{19} \mathrm{~cm}^{-3}$. For higher $\mathrm{Cl}$ concentrations the net donor concentration saturates and eventually begins to decrease in uniformly doped samples. ${ }^{3}$ Using planar-doping methods even higher free-electron concentrations have been achieved. ${ }^{4}$ In the case of $\mathrm{ZnSe}$, the interest has mainly been directed towards the problems with the $p$-type doping. In spite of numerous theoretical studies of doping in $\mathrm{ZnSe},{ }^{5-9}$ there exist only a few theoretical studies of the effect of chlorine in $\mathrm{ZnSe}$. In fact, to our knowledge, chlorine has been studied using modern electronic structure calculations only by Chadi. ${ }^{6}$ In his paper, Chadi shows that chlorine does not form a $D X$ center in $\mathrm{ZnSe}$.

We have made first-principles electronic-structure calculations of the formation energies and lattice relaxations for various chlorine-related defects in $\mathrm{ZnSe}$. The defects studied here include the most feasible defect complexes formed by a chlorine-impurity atom and a native point defect. In this paper only the most interesting Cl-related defects from an extensive set of studied defects are discussed in detail. The aim of the present paper is to shed light on the question of the actual fate of $\mathrm{Cl}$ in $\mathrm{ZnSe}$. Our calculations are based on the density-functional theory with the electron exchange correlation treated in the local-density approximation. ${ }^{10}$ In our calculations we have employed supercells containing 32 and 64 zinc-blende lattice sites. The Brillouin-zone sampling consists of a $2 \times 2 \times 2$ Monkhorst-Pack k-point mesh $^{11}$ in the case of the 32-atom supercell. For the larger supercell the $2 \times 2 \times 2$ Chadi-Cohen k-point mesh ${ }^{12}$ has been used. For the chlorine ion a Vanderbilt-type ultrasoft pseudopotential ${ }^{13}$ has been employed. The use of ultrasoft pseudopotentials decreases the kinetic energy cutoff needed to describe the heavily peaked electronic states of $\mathrm{Cl}$ and a good convergence is obtained already with a 27 Ry cutoff energy. Further computational details can be found in our earlier study of nitrogen-related defects in $\mathrm{ZnSe} .{ }^{14}$ The defect formation energies given in this work have been calculated using a standard method. ${ }^{14,15}$ We use neutralizing uniform background charge in order to avoid long-range Coulomb interactions between supercells. The energy bands of the defect supercells are aligned with those of the perfect lattice using an average-potential correction. ${ }^{15,20}$

The organization of the paper is as follows: In Sec. II a detailed analysis of possible isolated chlorine impurities at different lattice sites is given. The analysis of the defect complexes is given in Sec. III. Section IV summarizes the paper.

\section{ISOLATED CHLORINE IMPURITIES}

We have investigated all possible substitutional and interstitial sites for an isolated chlorine atom. For every site full ionic relaxation without any symmetry restrictions has been performed. The formation energies and ionization levels, if any, are given in Table I. The chemical potential for the chlorine ion has been taken from the chlorine dimer $\left(\mathrm{Cl}_{2}\right)$. The absolute values given in Table I are thus not unambiguous, but the differences between them are. In other words, the values cannot be used to estimate the solubility of chlorine to $\mathrm{ZnSe}$, but the numbers are intended to be used to predict the effect of incorporated chlorine. From the values listed in Table I it is evident that most of the incorporated chlorine ions occupy selenium sites. This is also the experi-

TABLE I. Formation energies for chlorine-impurity defects in $\mathrm{ZnSe}$. The formation energies under different growth conditions can be calculated by setting $0 \leqslant \lambda \leqslant 1$ so that the formation energies in $\mathrm{Zn}$-rich conditions correspond to $\lambda=0$. The heat of formation $(\Delta H)$ calculated for $\mathrm{ZnSe}$ is $1.5 \mathrm{eV}$. The positions of the ionization levels are given for the defects having states in the band gap. $\mu_{e}$ is the electron chemical potential. All the energies given are in $\mathrm{eV}$.

\begin{tabular}{lcc}
\hline \hline Defect & Formation energy & Ionization level \\
\hline $\mathrm{Cl}_{\mathrm{Se}}{ }^{1+}$ & $-1.13+\lambda \Delta H+\mu_{e}$ & \\
$\mathrm{Cl}_{\mathrm{Zn}}{ }^{1+}$ & $5.38-\lambda \Delta H+\mu_{e}$ & $(+/-) 0.04$ \\
$\mathrm{Cl}_{\mathrm{Zn}}{ }^{1-}$ & $5.46-\lambda \Delta H-\mu_{e}$ & \\
$\mathrm{Cl}_{i}{ }^{1+}\left(\mathrm{T}_{\mathrm{Zn}}\right)$ & $3.71+\mu_{e}$ & $(+/-) 0.02$ \\
$\mathrm{Cl}_{i}{ }^{1-}\left(\mathrm{T}_{\mathrm{Zn}}\right)$ & $3.75-\mu_{e}$ & \\
$\mathrm{Cl}_{i}{ }^{1+}\left(\mathrm{T}_{\mathrm{Se}}\right)$ & $2.01+\mu_{e}$ & $(+/-) 1.46$ \\
$\mathrm{Cl}_{i}{ }^{1-}\left(\mathrm{T}_{\mathrm{Se}}\right)$ & $4.92-\mu_{e}$ & \\
\hline \hline
\end{tabular}




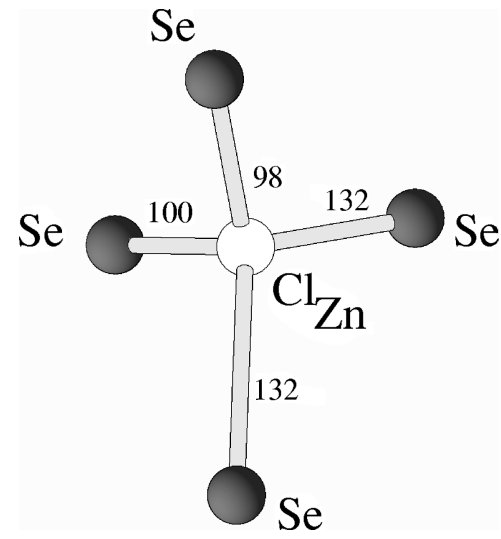

FIG. 1. Ionic structure of the negatively charged chlorine antisite $\left(\mathrm{Cl}_{\mathrm{Zn}}{ }^{-}\right)$defect. The bond lengths are given in percent of the ideal bond length.

mental observation, even in the case of $\mathrm{Cl}$-ion implanted $\mathrm{ZnSe}^{16}$

The substitutional chlorine (chlorine on the selenium site $\mathrm{Cl}_{\text {Se }}$ ) has only one stable charge state: the singly positive one. This is because the substitutional chlorine does not introduce any electronic states into the band gap. Thus, $\mathrm{Cl}_{\mathrm{Se}}$ is an efficient donor. The relaxations around the chlorine ion are very weak: the neighboring zinc ions move away from the chlorine site by $\sim 0.02 \AA$. The $T_{d}$ symmetry is conserved during the relaxation. We do not find any displacement of the chlorine ion away from the substitutional site observed for a significant fraction of chlorine ions by ion channeling techniques for samples doped using the ion implantation method. ${ }^{16}$ The formation energy of the substitutional chlorine is relatively low and therefore most of the incorporated chlorine atoms are expected to occupy the substitutional selenium sites.

The chlorine antisite $\mathrm{Cl}_{\mathrm{Zn}}$ has five possible charge states $(3+, 2+, 1+, 0$, and $1-)$, but it turns out that only the singly positive and singly negative ones become thermally occupied. The negative- $U$ transition between these states is located just above the valence band maximum. The formation energies for the chlorine antisites are so much higher than those for the substitutional chlorine that the creation of the chlorine antisite is unlikely during the crystal-growth process. The symmetry-breaking lattice relaxations shown in Fig. 1 are strong around the defect. The relaxation pushes two of the neighboring Se ions further away from the chlorine ion, whereas the other two Se ions remain approximately at the distance of the ideal-lattice bond length from the $\mathrm{Cl}$ ion. The chlorine impurity ion does not stay in the fourfold-coordinated zinc sublattice site.

Possible charge states for the interstitial chlorine in the tetrahedral site surrounded by zinc ions $\mathrm{Cl}_{i}\left(\mathrm{~T}_{\mathrm{Zn}}\right)$ are $1+, 0$, and $1-$. From them only the negative and the positive one can become thermally occupied. The negative- $U$ transition between these charge states is located just above the valenceband maximum. In the negative charge state the zinc ions neighboring the chlorine ion move away from the chlorine by $\sim 2 \%$ of the ideal (bond) length. When electrons are transferred away from the defect, the outwards relaxation weakens and finally for the $1+$ charge state a slight inwards relaxation takes place.
The only isolated Cl-impurity defect, which has states deep in the band gap is the chlorine interstitial in the tetrahedral selenium site $\left[\mathrm{Cl}_{i}\left(\mathrm{~T}_{\mathrm{Se}}\right)\right]$. Its possible charge states are the singly positive, the neutral, and the singly negative one. The neutral charge state is metastable and the negative- $U$ transition level between the positive and negative charge states is located $\sim 1.46 \mathrm{eV}$ above the valence-band maximum. Ionic relaxations of the surrounding lattice are extraordinary: the nearest-neighbor selenium ions move away from the chlorine ion while the next-nearest-neighbor zinc ions move towards it. As a result, in the positive charge state the chlorine ion bonds to three neighboring zinc ions and to one selenium ion. In the negative charge state the chlorine ion pushes all four selenium nearest-neighbor ions further away from itself and all the six next-nearest zinc ions are closer to the chlorine ion than any of the original nearest-neighbor selenium ions. These relaxation patterns reflect a strong ionic character in the interactions between the chlorine interstitial and the neighboring zinc and selenium ions.

\section{CHLORINE-IMPURITY NATIVE-DEFECT COMPLEXES}

According to the previous section, the formation energy for the substitutional chlorine donor is much lower than the formation energies of any possible compensating center formed by an isolated chlorine impurity. Therefore the compensation observed ${ }^{3}$ has to be due to native defects or due to complexes formed by the chlorine impurity bound to some native defect. Among the native defects in $n$-type $\mathrm{ZnSe}$ the doubly negative zinc vacancy has been shown to be energetically the most favorable one. ${ }^{14}$ Thus the compensation in $n$-type $\mathrm{ZnSe}$ is due to the formation of isolated zinc vacancies, to some other impurities, or to the presence of defect complexes. Recently, Saarinen et al. have identified zinc vacancies in $n$-type $\mathrm{ZnSe}: \mathrm{Cl}$ using positron annihilation experiments. ${ }^{17}$ The defect complex $\mathrm{Cl}_{\mathrm{Se}} \mathrm{V}_{\mathrm{Zn}}$ has been suggested to be formed in high-dose $\mathrm{Cl}$ implantation into ZnSe. ${ }^{16}$ We have studied possibilities for the creation of defect complexes formed by the zinc interstitial and by selenium or zinc vacancies with the substitutional or interstitial chlorine. Our results for the formation energies of the most feasible of such complexes are listed in Table II.

The stablest defect complex formed by the chlorine impurity and a native defect in $n$-type $\mathrm{ZnSe}$ is $\mathrm{Cl}_{\mathrm{Se}} \mathrm{V}_{\mathrm{Zn}}$. This nearest-neighbor pair is tightly bound: in the negative charge state the binding energy relative to the isolated $\mathrm{V}_{\mathrm{Zn}}{ }^{2-}$ and $\mathrm{Cl}_{\mathrm{Se}}{ }^{1+}$ defects is $1.44 \mathrm{eV}$. Although we do not find any other stable charge states for this defect complex, there is a possibility for the formation of a triply negative defect. This is because just above the conduction-band minimum there is an electronic state with localized character. The occupancy of that state could be possible under certain circumstances. As a matter of fact, the recently found emergence of a defect state as hydrostatic pressure is applied to $n$-type, chlorine-doped ZnSe (Ref. 18) is probably related to this state. The ionic relaxations around the Cl-impurity $\mathrm{Zn}$-vacancy pair shown in Fig. 2 conserve the $C_{3 v}$ symmetry of the defect. The negative charge state (favoring positron trapping at the defect) and the low formation energy of the $\mathrm{Cl}_{\mathrm{Se}} \mathrm{V}_{\mathrm{Zn}}$ complex give 
TABLE II. Formation energies of chlorine-impurity nativedefect complexes in $\mathrm{ZnSe}$. $\lambda=0$ corresponds to the Zn-rich conditions. The positions of the ionization levels are given for the defects having states in the band gap. The heat of formation $(\Delta H)$ calculated for $\mathrm{ZnSe}$ is $1.5 \mathrm{eV} . \mu_{e}$ is the electron chemical potential. All the energies given are in $\mathrm{eV}$.

\begin{tabular}{lcc}
\hline \hline Defect & Formation energy & Ionization levels \\
\hline$\left(\mathrm{Cl}_{\mathrm{Se}} \mathrm{V}_{\mathrm{Zn}}\right)^{1-}$ & $2.11-\mu_{e}$ & \\
$\left(\mathrm{Cl}_{\mathrm{Se}} \mathrm{Zn}_{i}\right)^{1+}$ & $0.73+\lambda \Delta H+\mu_{e}$ & \\
$\left(\mathrm{Cl}_{\mathrm{Se}} \mathrm{V}_{\mathrm{Se}}\right)^{3+}$ & $-0.82+2 \lambda \Delta H+3 \mu_{e}$ & $(3+/ 1+) 0.97$ \\
$\left(\mathrm{Cl}_{\mathrm{Se}} \mathrm{V}_{\mathrm{Se}}\right)^{1+}$ & $1.11+2 \lambda \Delta H+\mu_{e}$ & $(1+/ 1-) 1.41$ \\
$\left(\mathrm{Cl}_{\mathrm{Se}} \mathrm{V}_{\mathrm{Se}}\right)^{1-}$ & $3.92+2 \lambda \Delta H-\mu_{e}$ & \\
{$\left[\mathrm{Cl}_{i}\left(\mathrm{~T}_{\mathrm{Se}}\right) \mathrm{V}_{\mathrm{Zn}}\right]^{3+}$} & $2.03-\lambda \Delta H+3 \mu_{e}$ & $(3+/ 1+) 0.65$ \\
{$\left[\mathrm{Cl}_{i}\left(\mathrm{~T}_{\mathrm{Se}}\right) \mathrm{V}_{\mathrm{Zn}}\right]^{1+}$} & $3.32-\lambda \Delta H+\mu_{e}$ & $(1+/ 1-) 0.93$ \\
{$\left[\mathrm{Cl}_{i}\left(\mathrm{~T}_{\mathrm{Se}}\right) \mathrm{V}_{\mathrm{Zn}}\right]^{1-}$} & $5.18-\lambda \Delta H-\mu_{e}$ & \\
{$\left[\mathrm{Cl}_{\mathrm{Zn}} \mathrm{V}_{\mathrm{Se}}\right]^{1+}$} & $4.30+\mu_{e}$ & \\
\hline \hline
\end{tabular}

strong evidence that the zinc vacancy seen in positron annihilation measurements ${ }^{17}$ is due to this defect complex.

The lattice relaxations around the $\mathrm{Cl}_{\mathrm{Se}} \mathrm{Zn}_{i}$ defect in its only stable state, singly positive, are visualized in Fig 3. All the bonds around the chlorine ion, except that between the substitutional chlorine and a zinc ion away from the center, increase from the ideal-lattice bond length. The binding energy of the $\mathrm{Cl}_{\mathrm{Se}} \mathrm{Zn}_{i}$ defect is low, since both of its constituents are, in principle, positively charged. The effect of the substitutional chlorine near the zinc interstitial is, however, to pull the electronic state corresponding to the neutral charge state of $\mathrm{Zn}_{i}$ from the conduction band into the band gap. This state stabilizes the complex. Since the $\mathrm{Cl}_{S \mathrm{e}} \mathrm{Zn}_{i}$ defect is stable only in the positive charge state it cannot cause donor compensation.

The next-nearest-neighbor defect complex $\mathrm{Cl}_{\mathrm{Se}} \mathrm{V}_{\mathrm{Se}}$ has stable charge states ranging from $3+$ to $1-$. The triply posi-

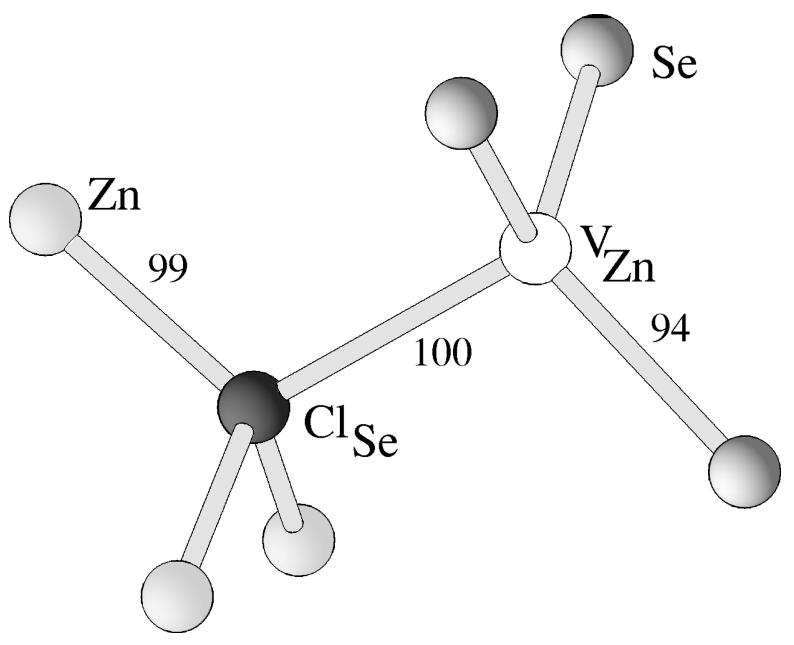

FIG. 2. Ionic structure of the $\mathrm{Cl}_{\mathrm{Se}} \mathrm{V}_{\mathrm{Zn}}$ defect complex. The bond lengths are given in percent of the ideal-lattice bond length. The center of the vacancy (white sphere) is defined as the position of a $\mathrm{Zn}$ ion in the perfect lattice and by thinking that ions far from the defect do not remarkably move from their ideal-lattice positions in the defect superlattice. The symmetry of the defect pair is conserved in relaxation.

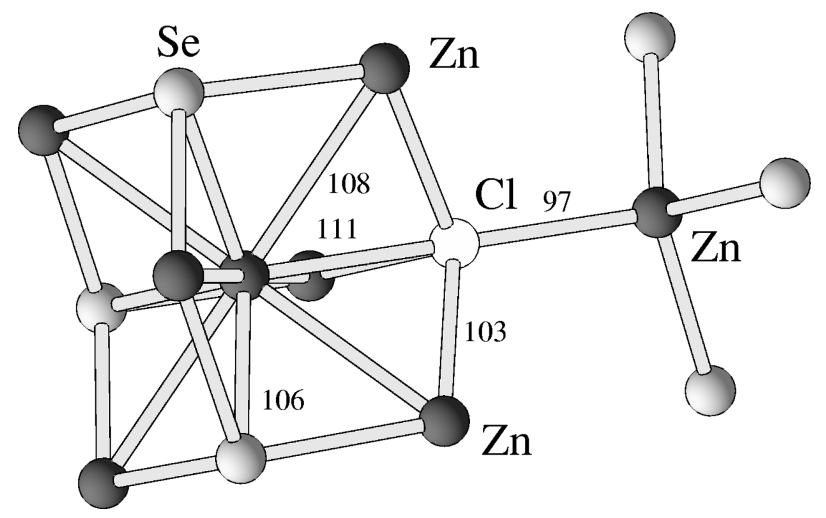

FIG. 3. Ionic structure of the $\mathrm{Cl}_{\mathrm{Se}} \mathrm{Zn}_{i}$ defect complex. The bond lengths are given in percent of the ideal-lattice bond length.

tive charge state is, however, unlikely to form since the binding energy is negative, indicating that the pair would easily break to separated $\mathrm{Cl}_{\mathrm{Se}}{ }^{1+}$ and $\mathrm{V}_{\mathrm{Se}}{ }^{2+}$ defects. In $n$-type $\mathrm{ZnSe}$, when the $\mathrm{Cl}_{\mathrm{Se}} \mathrm{V}_{\mathrm{Se}}$ defect complex is in a singlypositive or negative charge state, its creation from its isolated constituents is energetically favorable. The calculated binding energy for the singly positive charge state is $0.3 \mathrm{eV}$. The lattice relaxations around the Se vacancy are strong. Two of the neighboring zinc ions relax strongly towards the center of the vacancy in both of the positive and negative charge states, whereas the two other zinc ions relax outwards in the positive and inwards in the negative charge states. At the chlorine-impurity end of the defect three neighboring zinc ions stay almost at their ideal lattice sites and their relaxations do not depend strongly on the charge state. The detailed structures of defect in positive and negative charge states are visualized in Fig. 4. The formation energy for the selenium-vacancy-substitutional-chlorine pair is in any case so high that it is not a good candidate for the compensating center. Recent positron annihilation experiments by Saarinen et al. ${ }^{17}$ show, in good agreement with our results, that vacancy-type defects in $\mathrm{ZnSe}: \mathrm{Cl}$ are created in the zinc sublattice, not in the selenium sublattice.

The defect formed by a chlorine interstitial at the tetrahedral site surrounded by selenium ions $\mathrm{Cl}_{i}\left(\mathrm{~T}_{\mathrm{Se}}\right)$ and a zinc vacancy located at two bond lengths away from the interstitial is depicted in Fig. 5. The ionic relaxations are very strong for this defect complex. Interstitial chlorine and the selenium atom between $\mathrm{Cl}_{i}$ and $\mathrm{Zn}$ vacancy move as a pair towards the vacancy, so that in the relaxed structure there are in fact two small vacancies $\left(\mathrm{V}_{\mathrm{Se}}\right.$ and $\left.\mathrm{V}_{\mathrm{Zn}}\right)$; halfway between them is a Se-Cl pair. The binding energy of this quite distant pair is surprisingly high: $1.3 \mathrm{eV}$ in the negative charge state. The high binding energy makes this defect complex a promising candidate for the compensating center in $n$-type $\mathrm{ZnSe}$.

The chlorine-antisite-selenium-vacancy pair is in fact a metastable state of the substitutional-chlorine-zinc-vacancy pair. The barrier towards the re-creation of the $\mathrm{Cl}_{\mathrm{Se}} \mathrm{V}_{\mathrm{Zn}}$ defect is low even for the positive charge state. The chlorine ion does not stay at a fourfold coordinated zinc sublattice site, but moves towards the selenium vacancy by about $50 \%$ of the ideal-lattice bond length. A similar metastable behavior connected to the change in the charge state of a complex has previously been found even in an even more pronounced way for defect complexes in GaAs. ${ }^{19,20}$ 


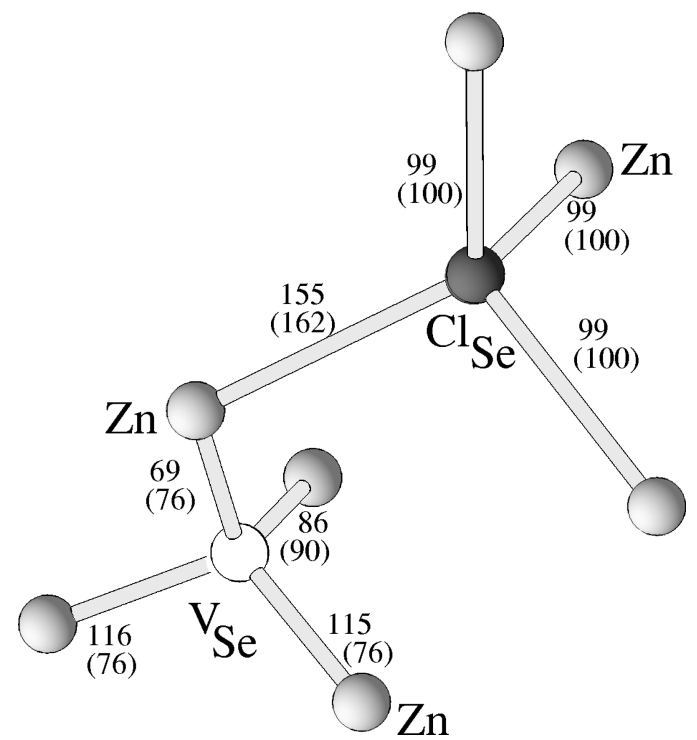

FIG. 4. Ionic structure of the $\mathrm{Cl}_{\mathrm{Se}} \mathrm{V}_{\mathrm{Se}}$ defect pair. The center of the vacancy (white sphere) is defined as the position of a $\mathrm{Zn}$ ion in the perfect lattice and by assuming that ions far from the defect do not move from their ideal-lattice positions in the defect superlattice. The distances of the $\mathrm{Zn}$ ions (light gray spheres) from the vacancy center and from the $\mathrm{Cl}$ ion (dark gray sphere) are given in percent of the bond length of the ideal lattice for the positive (upper numbers) and for the negative (numbers in parenthesis) charge state.

The formation energy of the substitutional chlorine impurity attains its lowest values if the material is grown under Zn-rich conditions, whereas the formation energy of the most probable compensating center, i.e., that of $\mathrm{Cl}_{\mathrm{Se}} \mathrm{V}_{\mathrm{Zn}}$, does not depend on the stoichiometry. Thus, the increase in the parameter $\lambda$, dictating the stoichiometry, favors the generation of the compensating centers instead of the formation of substitutional chlorine donors. Therefore the best growth condi-

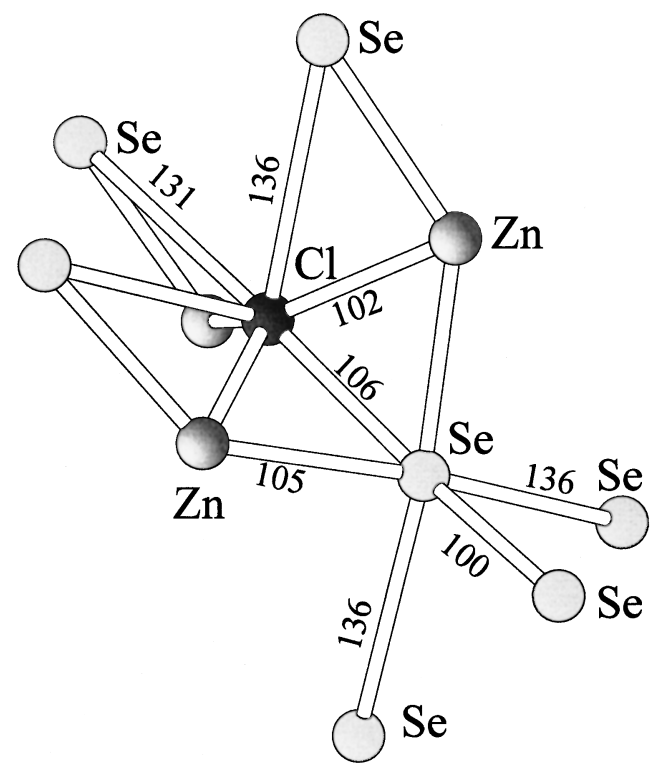

FIG. 5. Ionic structure of the $\mathrm{Cl}_{i} \mathrm{~V}_{\mathrm{Zn}}$ defect pair. To clarify the figure, only the nearest neighbors of the chlorine ion and the $\mathrm{Zn}$ vacancy are shown. The interatomic distances are given in percent of the bond length of the ideal lattice.

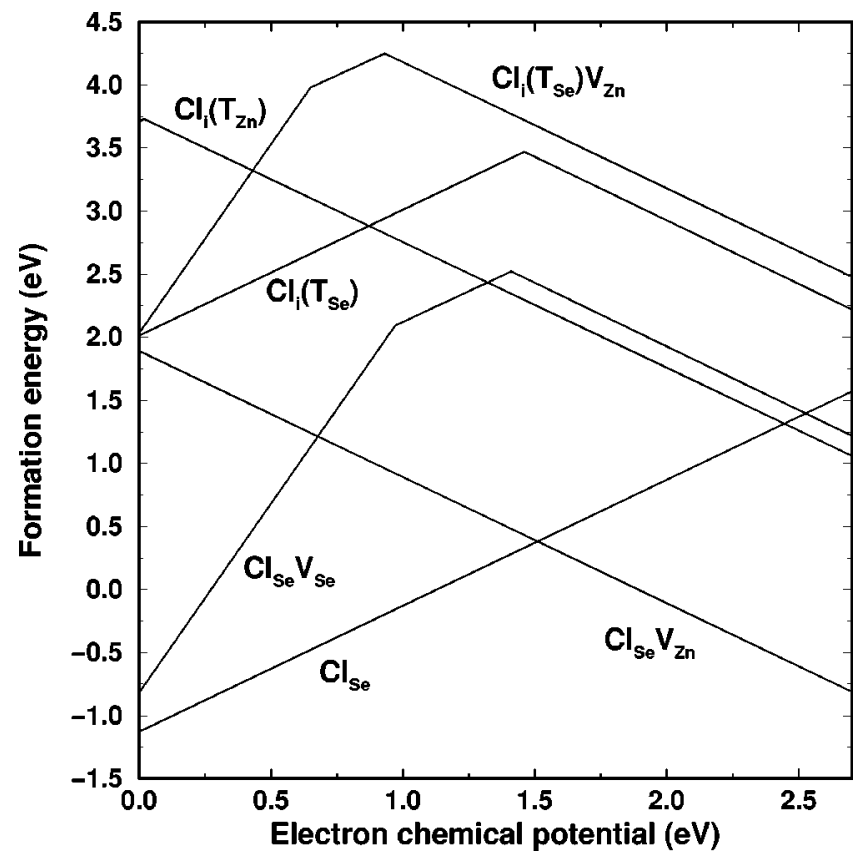

FIG. 6. Formation energies for the chlorine-related defects with the lowest formation energy values under Zn-rich conditions.

tions for obtaining the highest effective donor concentrations are $\mathrm{Zn}$-rich conditions. Figure 6 shows, as a function of the electron chemical potential, the formation energies for those chlorine-related defects that have the lowest formation energies in $\mathrm{ZnSe}$ grown under $\mathrm{Zn}$-rich conditions $(\lambda=0)$. As the Fermi level rises, for example, due to chlorine doping, the formation energy of the donor impurity increases, and the formation of compensating defect complexes becomes more favored. Thus these chlorine-related defect complexes are formed and further chlorine incorporated into $\mathrm{ZnSe}$ decreases the doping efficiency.

\section{CONCLUSIONS}

We have shown that most of the chlorine incorporated in ZnSe occupies selenium lattice sites indicating an effective donor doping. The chlorine-induced lattice relaxations are shown to be generally small, but in the case of defect complexes the softness of the $\mathrm{ZnSe}$ lattice manifests itself as large distortions. Our calculations show that the donor compensation observed in $n$-type chlorine-doped $\mathrm{ZnSe}$ is due to the creation of defect complexes. The most promising candidate for the compensating center is the substitutionalchlorine-zinc-vacancy complex. Since in every good candidate for the compensating center there is a zinc vacancy as a part of the complex or as an isolated defect, these results are in excellent agreement with the recent positron annihilation measurements by Saarinen et al. ${ }^{17}$

\section{ACKNOWLEDGMENTS}

The authors wish to thank P. Hautojärvi, K. Saarinen, K. Laasonen, and T. Mattila for many valuable discussions. This work has been supported by the Academy of Finland through a MATRA grant. We also acknowledge the generous computing resources of the Center for the Scientific Computing (CSC), Espoo, Finland. 
*Electronic address: Sami.Poykko@hut.fi

†Electronic address: Martti.Puska@hut.fi

†Electronic address: Risto.Nieminen@hut.fi

${ }^{1}$ R. M. Park, M. B. Troffer, J. M. DePuydt, and M. A. Haase, Appl. Phys. Lett. 57, 2127 (1990).

${ }^{2}$ K. Ohkawa, T. Karasawa, and T. J. Mitsuyo, Cryst. Growth 111, 797 (1991).

${ }^{3}$ K. Ohkawa, T. Mitsuyo, and O. Yamazaki, J. Appl. Phys. 62, 3216 (1987).

${ }^{4}$ Z. Zhu, H. Mori, and T. Yao, Appl. Phys. Lett. 61, 2811 (1992).

${ }^{5}$ D. B. Laks, C. G. Van de Walle, G. F. Neumark, P. E. Blöchl, and S. T. Pantelides, Phys. Rev. B 45, 10965 (1992).

${ }^{6}$ D. J. Chadi, Phys. Rev. Lett. 72, 534 (1994).

${ }^{7}$ Byoung-Ho Cheong, C. H. Park, and K. J. Chang, Phys. Rev. B 51, 10610 (1995).

${ }^{8}$ C. H. Park and D. J. Chadi, Phys. Rev. Lett. 75, 1134 (1995).

${ }^{9}$ K. W. Kwak, R. D. King-Smith, and D. Vanderbilt, Phys. Rev. B 48, 17827 (1993).

${ }^{10}$ D. M. Ceperley and B. J. Alder, Phys. Rev. Lett. 45, 566 (1980);
J. Perdew and A. Zunger, Phys. Rev. B 23, 5048 (1981).

${ }^{11}$ H. J. Monkhorst and J. D. Pack, Phys. Rev. B 13, 5188 (1976).

${ }^{12}$ D. J. Chadi and M. L. Cohen, Phys. Rev. B 8, 5747 (1973).

${ }^{13}$ D. Vanderbilt, Phys. Rev. B 41, 7892 (1990); K. Laasonen, A. Pasquarello, R. Car, C. Lee, and D. Vanderbilt, ibid. 47, 10142 (1993).

${ }^{14}$ S. Pöykkö, M. J. Puska, and R. M. Nieminen, Phys. Rev. B (to be published 15 May 1998).

${ }^{15}$ A. Garcia and J. E. Northrup, Phys. Rev. Lett. 74, 1131 (1995).

${ }^{16}$ K. M. Yu, J. W. Ager III, E. D. Bourret, and W. Walukiewicz, J. Appl. Phys. 75, 1378 (1995).

${ }^{17}$ K. Saarinen, T. Laine, K. Skog, J. Mäkinen, P. Hautojärvi, K. Rakennus, P. Uusimaa, A. Salokatve, and M. Pessa, Phys. Rev. Lett. 77, 3407 (1996).

${ }^{18}$ T. M. Ritter, B. A. Weinstein, R. M. Park, and M. C. Tamargo, Phys. Rev. Lett. 76, 964 (1996).

${ }^{19}$ G. A. Baraff and M. Schlüter, Phys. Rev. Lett. 55, 2340 (1985).

${ }^{20}$ S. Pöykkö, M. J. Puska, M. Alatalo, and R. M. Nieminen, Phys. Rev. B 54, 7909 (1996). 